|IIIIIIIIIIIIIIIIIIIIIIIIIIIIIIIIII

Original Article

IIIIIIIIIIIIIIIIIIIIIIIIIIIIIIIIII

\title{
Compatibility of entomopathogenic fungi with insecticides and their efficacy for IPM of Bemisia tabaci in cotton
}

\author{
Satish Kumar Sain, ${ }^{1, *}$ Dilip Monga, ${ }^{1}$ Rishi Kumar, ${ }^{1}$ Dipak T. Nagrale, ${ }^{2}$ \\ Neelakanth S. Hiremani ${ }^{2}$ and Sandhya KranthI ${ }^{2}$ \\ ${ }^{1}$ ICAR-Central Institute for Cotton Research, Regional Station, Sirsa (Haryana), India \\ ${ }^{2}$ ICAR- Central Institute for Cotton Research, Nagpur, (Maharashtra), India
}

(Received October 5, 2018; Accepted January 22, 2019)

\begin{abstract}
Bemisia tabaci, a vector of cotton leaf curl virus disease, is among the most devastating pests causing huge economic losses due to reduced cotton yield and quality. The excessive use of chemical pesticides causes insecticide resistance. Entomopathogenic fungi (EPFs) have a role as mycoinsecticides. The combined use of these insecticides is a promising pest-control option to minimize adverse chemical effects. Thus, we have evaluated $10 \mathrm{EPFs}$ under polyhouse conditions for their virulence against whitefly nymphs and their compatibility with chemical and botanical insecticides. The highest overall biological efficacy index was recorded with Ij-102, followed by Bb-4511, and Ij-089. An in vitro compatibility study was conducted to evaluate the effect of botanical and chemical pesticides on mycelial growth and spore production using the poisoned food technique. The effect of pesticides on the reduction of mycelial growth and conidial production ranged from -169 to $94.1 \%$ and -25.6 to $87.6 \%$, respectively. However, Ij-089, Ij-102, Ma-1299, and Bb-4511 were found to be the most compatible with the chemical and botanicals evaluated. Comparatively, spiromesifen, diafenthiuron, buprofezin, pyriproxyfen, and flonicamid were more compatible with EPFs at half doses, as compared to the other chemical pesticides, namely imidacloprid, fipronil, profenophos, and triazophos. These results might provide the basis for future work and indicate that applications of EPFs showing the best virulence and compatibility have the maximum likelihood for the management of $B$. tabaci in the field in an integrated pest management system. (c) Pesticide Science Society of Japan

Keywords: Bemisia tabaci, entomopathogenic fungi, biocontrol efficacy, insecticides compatibility, insecticide resistance management, integrated pest management.

Electronic supplementary material: The online version of this article contains supplementary material (Supplemental Tables S1-S6), which is available at http://www.jstage.jst.go.jp/browse/jpestics/
\end{abstract}

\section{Introduction}

Cotton (Gossypium spp.), known as "white gold", is one of the world's most commercially important and natural textile fiber crops and a significant contributor of oil seeds. The cotton crop in India was cultivated on 12.9 million ha during Kharif season, from April to October 2017, with production of 37.7 million bales and productivity of $524 \mathrm{~kg}$ lint/ha. It was grown in India's North Zone on 1.54 million ha. ${ }^{1)}$ Among several insect pests, the whitefly Bemisia tabaci (Gennadius) (Hemiptera: Aleyrodidae), a vector of cotton leaf curl diseases (CLCuD), is the most devastating and serious problem, inflicting huge economic losses due to reduced cotton yields and quality. The whitefly is highly polyphagous, invading more than 900 wild and cultivated species ${ }^{2)}$ and transmitting more than 110 plant viruses worldwide. ${ }^{3)}$ In

\footnotetext{
* To whom correspondence should be addressed.

E-mail: sain.skumar@gmail.com

Published online March 15, 2019

(c) Pesticide Science Society of Japan
}

the past, five outbreaks of whiteflies have been noticed in different cotton-growing states in India. In 2015-2016, a severe outbreak of whiteflies occurred in the north cotton growing zone of India, causing losses of up to $50-60 \%$ of the crop. ${ }^{4)}$ Damage results from direct feeding that reduces the yield. ${ }^{5)}$ Large amounts of honeydew excreted by the insect encourage the development of black sooty mold on leaves. $\left.{ }^{6}\right)$ The direct shading of leaves by the powdery coating has been reported to reduce the photosynthetic capabilities of crop plants, resulting in economic loss. ${ }^{7)}$ The greatest economic threat is from the transmission of $\mathrm{CLCuD}$ viruses, which causes yield losses of up to-81.4-88.4\% in all northern cotton-growing areas of India. ${ }^{8)}$ In a recent study, three $B$. tabaci biotypes were recorded in India, including AsiaII-7 in Pusa-Delhi, Asia-I in southern and central India, and Asia-II-1 in northern India. ${ }^{9)}$

Presently, there is no source of absolute resistance against $\mathrm{CLCuD}$ and its vector $B$. tabaci in cotton varieties and almost all of the cultivated Bt and non-Bt cotton hybrids, including about 250 new Bt cotton hybrids approved for cultivation in North India. ${ }^{10)}$ The urge to obtain greater yields and quality cotton is 
encouraging farmers to apply prophylactic sprays of pesticides to manage both whiteflies and CLCuD. Thirty-five insecticides have been registered for whitefly management in India. It has acquired resistance to many insecticide classes. ${ }^{9,11-14)}$ Of the 109 pesticide products registered so far for managing cotton pests in India, only one mycoinsecticide (Lecanicillium lecanii R. Zare \& W. Gams) (Hypocreales: Clavicipitaceae) is recommended for whitefly management in cotton. ${ }^{15)}$ Several recent studies have demonstrated the different modes of action of mycoinsecticides against insect pests, such as endophytes, plant disease antagonism, plant growth promotion, and rhizosphere colonization. Consequently, the present situation is emphasizing the need for research to find an environmentally friendly and sustainable method of managing this tedious pest. More than 20 species of entomopathogenic fungi (EPFs) are known to infect whiteflies, which could be ecologically compatible and a good insecticideresistance management alternative for whiteflies. ${ }^{16-19)}$ Additionally, about 170 EPF strains have been commercialized as biocontrol agents, and the majority of them have been developed from Ascomycota Beauveria spp. (Bals-Criv.) Vuill. (Hypocreales: Clavicipitaceae) and Metarhizium spp. (Metschn.) Sorok. (Hypocreales: Clavicipitaceae). ${ }^{20,21)}$ Additionally, the combined use of mycoinsecticides and the full or reduced dose of chemical insecticides is a promising pest-control option for minimizing adverse chemical effects. Moreover, effective EPFs and selective insecticides may act synergistically to increase the efficiency of the control, allowing lower doses of insecticides and the preservation of natural enemies, while minimizing environmental pollution and decreasing the likelihood of developing resistance to either agent. ${ }^{22)}$ The potential inhibitory effects of pesticides on the germination and mycelial growth of EPFs often vary among fungal species and strains. ${ }^{23)}$ By contrast, the use of incompatible insecticides may inhibit the growth and reproduction of the pathogens and adversely affect integrated pest management (IPM) ${ }^{24,25)}$ Therefore, fungal genotypes compatible with particular pesticides can be identified and manipulated to develop a suitable IPM or insecticide resistance management (IRM) program. However, little information is available on their relative compatibility with IPM components.

Hence, the present investigation was conducted to evaluate EPFs for their relative virulence against whiteflies and their compatibility with various chemical and botanical insecticides to utilize their full biocontrol potential in IPM technology for successful adoption in the field. It was presumed that this study could ultimately help to identify the most virulent and IPMcompatible EPFs. It was also presumed that this study might provide the basis for future work on the development of an ecocompatible and effective mycopesticide to strengthen the current IPM/IRM system for managing the CLCuD vector B. tabaci in cotton.

\section{Materials and Methods}

The works described below were conducted during the 2016-2017 and 2017-2018 crop years at ICAR's Central Insti- tute for Cotton Research, Regional Station Sirsa (Haryana) $\left(29^{\circ} 32^{\prime} 36.1^{\prime \prime} \mathrm{N} 75^{\circ} 02^{\prime} 18.8^{\prime \prime} \mathrm{E}\right)$.

\section{Fungal strains}

The EPFs used in this study were Beauveria bassiana $(\mathrm{Bb})$ NAIMCC 0409 and Metarrhizium anisopliae (Ma) NAIMCC 1299, procured from the National Agriculturally Important Microbial Culture Collection (NAIMCC), Mau, Uttar Pradesh (India); B. bassiana MTCC 4511, MTCC-4543, MTCC 4565, and MTCC 6097 from the Microbial Type Culture Collection (MTCC), Chandigarh (India); and four indigenous cultures of Fusarium moniliforme (Fm) and Isaria javanica (Ij) isolated from $B$. tabaci cadavers. The newly isolated EPF cultures were identified on the basis of both morphological ${ }^{26)}$ and molecular characterizations (Supplemental Table S1). All fungal strains/ isolates were cultured and preserved in the laboratory on Sabouraud Dextrose Yeast Agar (HiMedia) amended with 0.2\% yeast (SDYA) and streptomycin sulfate $(20 \mu \mathrm{g} / \mathrm{L})$ in test tubes/ Petri plates. The provisional species were identified (Fm-083, Ij089, Ij-099, and Ij-102) by morphology, and it was corroborated later by genetic analysis after extracting the DNA of the monosporic cultures using PowerLyzer ${ }^{\circledR}$ UltraClean ${ }^{\circledR}$ Microbial DNA Isolation Kit (MO BIO Laboratories, Inc., USA). Polymerase chain reactions (PCRs) were carried out in a thermal cycler to amplify and sequence the internal-transcribed-spacer rDNA region. The genomic DNAs were amplified using universal forward primer (ITS1) 5' -TCC GTA GGT GAA CCT GCG G-3' and reverse primer (ITS4) 5' -TCC TCC GCT TAT TGA TAT GC$3^{\prime} .{ }^{27)}$ The amplicons of isolates separated on agarose gel were visualized in a UV transilluminator. The purification of amplicons of samples was done by using ExoSAP-IT PCR Cleanup (Affymetrix-USB, USA). The samples were sequenced by Eurofins Genomics India Pvt. Ltd., India. The chromatogram quality of sequences was checked with Applied Biosystems Sequence scanner v 1.0 software. The contigs were formed using the CAP3 sequence assembly program ${ }^{28)}$ from respective forward and reverse sequences of isolates. The contiguous sequences of fungal isolates were compared with those available in the database of the National Center for Biotechnology Information (NCBI) with a BLASTn similarity search. The choice of these fungal strains was based on their preliminary polyhouse bioassay conducted for whitefly nymphal mortality during 2016-2017 at the ICARCICR Regional Station, Sirsa.

\section{Insecticides}

Nine synthetic chemical pesticides and three botanicals were procured from the local market and used in these experiments. The insecticides were tested for fungal compatibility at two doses by in vitro bioassay: the average concentration recommended for field application on cotton for whitefly management (100\%, full dose) and 50\% (half dose) of the average. The insecticide formulations used for compatibility studies were: neem oil $0.03 \%(5 \mathrm{~mL} / \mathrm{L})$, pongamia oil $(5 \mathrm{~mL} / \mathrm{L})$, castor oil (10 mL/L), spiromesifen $22.9 \%$ SC ( $1 \mathrm{~mL} / \mathrm{L})$, flonicamid $50 \%$ 
WG $(0.4 \mathrm{~g} / \mathrm{L})$, diafenthiuron $50 \%$ WG $(1 \mathrm{~g} / \mathrm{L})$, buprofezin $25 \%$ SC $(1.6 \mathrm{~mL} / \mathrm{L})$, pyriproxyfen $10 \% \mathrm{EC}(2.5 \mathrm{~mL} / \mathrm{L})$, profenophos $50 \%$ EC $(2 \mathrm{~mL} / \mathrm{L})$, triazophos $40 \%$ EC $(3 \mathrm{~mL} / \mathrm{L})$, imidacloprid $70 \% \mathrm{WG}$, and fipronil 5\% EC (2 mL/L) (Supplemental Table S2).

\section{Screening of entomopathogens for their virulence}

\subsection{Polyhouse screening of EPFs}

All EPFs were cultured at the Plant Pathology Laboratory, ICAR-CICR Regional Station, Sirsa, Haryana (India). The fungal cultures were inoculated and incubated on SDYA amended with streptomycin sulfate $(20 \mu \mathrm{g} / \mathrm{L})$ in sterilized Petri plates for $7-10$ days at $25 \pm 2{ }^{\circ} \mathrm{C}$ in the dark. The mycelial growth of each fungal culture, as well as its sporulation, was estimated at 10 days after inoculation (DAI) and their cumulative deficiencies compared.

The spore concentration was recorded from each actively growing fungal strain on SDYA by using a $5 \mathrm{~mm}$ disc 10 DAI. Conidia were harvested from each Petri plate by flooding media with sterile $0.01 \%(\mathrm{v} / \mathrm{v})$ Tween $80^{\mathrm{TM}}$ (PEG-80 polyoxyethylene sorbitan monolaurate; HiMedia) and stirring with a glass rod. The suspension was vigorously vortexed for $2 \mathrm{~min}$ and filtered through four layers of sterilized nylon cheesecloth. The filtered suspension $(10 \mathrm{~mL})$ was agitated again before spray application, and the conidial concentration of the stock was estimated with an improved Neubauer hemocytometer at $400 \times$ magnification. The conidial suspension was then diluted to get a series of concentrations between $10^{4}$ and $10^{8}$. In all bioassay tests, conidial inocula $\left(1 \times 10^{7}\right.$ conidia $\left./ \mathrm{mL}\right)$ harvested from 10 -day-old cultures and suspended in $0.01 \%(\mathrm{v} / \mathrm{v})$ surfactant (Tween 80, HiMedia) were utilized. The viability of the conidia from each isolate and the concentrations used in the tests were determined after $24 \mathrm{hr}^{19)}$ This germination test was repeated for each stock suspension to maintain the constancy of the viability assessments. The viability of conidia ( $>95 \%$ germination) $24 \mathrm{hr}$ after incubation on SDYA medium (HiMedia) was confirmed before the onset of bioassay.

The virulence of EPFs was evaluated using a new modified polyhouse bioassay method described by Sain et al. ${ }^{29)}$ In this, one-month-old potted cotton plants with 4-5 fully expanded leaves were kept inside a whitefly-rearing polyhouse containing whitefly-infested plants (50-60 whiteflies/leaf) for egg laying. One day post egg laying, whitefly adults were gently removed from the potted plants using the air pressure of a commercial hand sprayer, and the potted plants were transferred to another net house aseptically for the next 10 days. Subsequently, 10 days post egg laying, the number of nymphs (40-50 nymphs/ leaf) was recorded before inoculation and marked on the abaxial surface of the leaf with a waterproof marker. A freshly prepared conidial suspension $\left(1 \times 10^{7}\right.$ conidia/mL) of EPFs was applied to the abaxial surface of leaves containing nymphs with a commercial handheld sprayer at a volume of $\sim 10 \mathrm{~mL} /$ plant $(\sim 2 \mathrm{~mL}$ / leaf) in a polyhouse maintained at $75 \pm 2 \% \mathrm{RH} 30 \pm 2^{\circ} \mathrm{C}$. In the control treatment, potted plant leaves were sprayed with $0.01 \%$ Tween 80 solution only. One replicate comprised three potted plants with three leaves on each plant. Mortality was recorded using a $20 \times$ hand magnifying lens at 3, 5, and 7 DAI. Whitefly nymphs that were opaque or greenish white and shiny with brownish eyes or with visible honeydew droplets appearing on their excretions were considered alive, while nymphal bodies that were yellowish-brown, matted, and shriveled were considered dead. Abbott's correction formula was used to correct the control mortality ${ }^{30)}$ before subjecting mortality data to analysis of variance.

To select the best EPFs for their potential bioefficacy, the overall bioefficacy index (BI) was compared using the modified formula $\mathrm{BI}=37 \times(\mathrm{MG})+13 \times(\mathrm{SP})+50 \times(\mathrm{MO})$ of Sain et al., ${ }^{29)}$ where $\mathrm{MG}=$ mycelial growth in $\mathrm{mm}$ on the tenth day, $\mathrm{SP}=$ conidial production $/ \mathrm{mL}\left(1 \times 10^{8}\right)$ on the tenth day, and $\mathrm{MO}=$ nymphal mortality at $7 \mathrm{DAI}$.

\subsection{Log dose probit analysis}

The lethal concentration of selected EPFs was determined on whitefly nymphs using five concentrations from $10^{4}$ to $10^{8}$ conidia/mL in three replicates. The new modified polyhouse bioassay method ${ }^{30)}$ was used. Mortality was recorded using a 20X hand magnifying lens at 5 DAI. Abbott's correction formula was used to correct the control mortality. ${ }^{28)}$ Data of selected EPFs from three replications of five concentrations were pooled, Excel was used for probit analysis, ${ }^{31)}$ and the lethal concentrations for $50 \%$ mortality $\left(\mathrm{LC}_{50}\right)$ and $90 \%$ mortality $\left(\mathrm{LC}_{90}\right)$ were calculated, including their $95 \%$ fiducial limits.

\section{Compatibility study}

\subsection{Mycelial growth inhibition}

All 10 EPFs were evaluated for their compatibility with the nine chemicals and three botanical pesticides recommended for whitefly management in North India (Supplemental Table S2). The in vitro compatibility of these compounds was determined using the recommended dose and half of the recommended dose by the poison food technique. ${ }^{32)}$ To get the desired concentration of each insecticide, the recommended field application rate/dose as well as its half dose was added to the melted SDYA medium $(100 \mathrm{~mL})$ in a flask aseptically and mixed thoroughly before solidification (medium temperature $48^{\circ} \mathrm{C}$ ) to get the desired concentration. ${ }^{33)}$ About $20 \mathrm{~mL}$ of medium was then poured equally into $9 \mathrm{~cm}$ diameter sterile Petri plates and allowed to solidify in a laminar air flow chamber. The Petri plate containing the desired pesticide-poisoned medium was inoculated aseptically and separately by transferring a five mm diameter culture disc into the center of each Petri plate. The culture disc was cut using a sterile cork borer from 10-day-old EPFs grown on SDYA. After inoculation, Petri plates were sealed with parafilm and incubated at $28 \pm 2{ }^{\circ} \mathrm{C}$ and $80 \pm 5 \%$ relative humidity for a 12-hr photoperiod. For each treatment, three replications were maintained. Pesticide-unamended SDYA media served as the control treatment for comparison under the same conditions.

The radial growth of each individual treatment, including each EPF, was measured at seven DAI and compared with a standard check. The percent of growth inhibition of each EPF over the untreated check was estimated separately for the re- 
spective insecticides by using the following formula: $\mathrm{I}=\mathrm{C}-\mathrm{T}$ / $\mathrm{C} \times 100$, where $\mathrm{I}=$ percent growth inhibition, $\mathrm{C}=$ colony diameter in the control, $\mathrm{T}=$ colony diameter with treatment.

\subsection{Inhibition of spore production}

In addition to the mycelial growth measurement, to estimate the effect of chemical and botanical pesticides on the spore production of each EPF, the conidial production was enumerated. The spore concentration was recorded by using a $5 \mathrm{~mm}$ disc of SDYA containing each dose of the above-mentioned 12 pesticides and inoculated with individual EPFs, separately. The spore concentration was recorded at $10 \mathrm{DAI}$ and compared with that in unamended SDYA media. The toxicities of chemicals and botanicals against EPF were calculated using the formula of Alves and Lecuona $^{34)}: \mathrm{T}=[20(\mathrm{VG})+80(\mathrm{ESP})] / 100$. In this formula, values of vegetative growth (VG) and sporulation (ESP) were given in relation to control (100\%), where toxicity grades were considered to be as follows: $\mathrm{T}=0$ to 30 (very toxic), 31 to 45 (toxic), 46 to 60 (moderately toxic), and $>60$ (compatible).

\section{Statistical analysis}

All experiments were carried out in replicated randomized complete block design (RCBD) and factorial RCBD. Abbott's correction formula was used to correct the control mortality ${ }^{30)}$ before subjecting mortality data to analysis of variance (ANOVA). Abbott's corrected mortality $=((\%$ mortality in treatment $-\%$ mortality in control $) /(100-\%$ mortality in control $)) \times 100$.

Data of selected EPFs from three replications of four concentrations were pooled and subjected to Probit analysis ${ }^{31)}$ and the lethal concentrations for 50\% mortality $\left(\mathrm{LC}_{50}\right)$ and $90 \%$ mortality $\left(\mathrm{LC}_{90}\right)$ calculated, including their $95 \%$ fiducial limits. The statistical analyses for compatibility experiments were performed using OP Stats. ${ }^{35)}$ Means were also separated using T-test at a 5\% level of significance.

\section{Results and Discussion}

\section{Screening of entomopathogens for their virulence}

\subsection{Polyhouse screening of EPFs}

The sequences generated in this study for local and new EPFs were submitted to GenBank (accession numbers MG976231 for Fusarium moniliforme (Fm) Fm-083, MG976232 for Isaria javanica (Ij), and Ij-0089 and MG976234 for Ij-102). Among the 10 EPFs, the highest mycelial growth was recorded in $\mathrm{Ij}-089$, followed by Ij-102 and Fm 083. However, the highest spore count $\left(10^{8} / \mathrm{mL}\right)$ was recorded in $B$. bassiana $(\mathrm{Bb}) \mathrm{Bb}-409, \mathrm{Bb}-$ 4511 , and Fm-083. The corrected nymphal mortality recorded at seven DAI was significantly highest ( $p$-value $<5 \%$ ) in Bb-4511 (95.1\%), followed by Bb-4565 (89.9\%) and M. anisopliae (Ma) Ma-1299 (86.7\%) at $33.7-26.7^{\circ} \mathrm{C}$ maximum and minimum temperature and $80.3-68.4 \% \mathrm{RH}$ with suspension of $1 \times 10^{7}$ conidia/ $\mathrm{mL}$. As compared to the fungal strains obtained from the culture collection centers, the local isolates showed faster and higher mycelial growth. However, based on overall biological efficacy, the EPFs that performed best were found to be Ij-102 (70.9\%), followed by Bb-4511 (70.1\%) and Ij-089 (69.5\%) (Table 1).

Hence, we proposed that the overall biocontrol potential of a fungal strain should be based not only on its virulence, but on its bioefficacy, including factors such as mycelial growth, sporulation, and mortality. Several other researchers have previously reported that biopesticides provide good control of whiteflies, both in the greenhouse and the field. B. bassiana caused mortality from 76.7 to $91.6 \%$ of whitefly B. tabaci nymphs (Faria and Wraight $)^{20)}$ and up to $100 \%$ adults at $1 \mathrm{mg} / \mathrm{mL}^{36)}$ Similarly, $V$. lecanii at $0.25 \times 10^{6}$ and $3.2 \times 10^{6}$ conidia/mL have been reported to cause $92-100 \%$ nymphal mortality of B. tabaci. ${ }^{37)}$ Three different products of Metarrhizium have also been found to reduce the population of nymphs and adults, ranging from $85.8-92.7 \%$

Table 1. Whitefly nymphal mortality, mycelia growth, spore production and biological efficacy index of selected EPFs. ${ }^{a)}$

\begin{tabular}{|c|c|c|c|c|c|c|}
\hline \multirow{2}{*}{ Treatments } & \multirow{2}{*}{ Mycelial growth ${ }^{b)}$} & \multirow{2}{*}{ Spore $/ \mathrm{mL}\left(10^{8}\right)^{c)}$} & \multicolumn{3}{|c|}{ Percent corrected mortality over control $\left(1 \times 10^{6}\right)^{d)}$} & \multirow{2}{*}{$\begin{array}{c}\text { Biological efficacy } \\
\text { index }^{e)}\end{array}$} \\
\hline & & & $3 \mathrm{DAI}$ & $5 \mathrm{DAI}$ & $7 \mathrm{DAI}$ & \\
\hline I. javanica-089 & 81.0 & 5.47 & $42.4(40.6)$ & $73.9(59.3)$ & $77.6(61.8)$ & 69.5 \\
\hline I. javanica-102 & 80.1 & 5.66 & $38.6(38.4)$ & $61.7(51.8)$ & $81.0(64.2)$ & 70.9 \\
\hline F. moniliforme- 083 & 76.5 & 6.33 & $42.0(40.4)$ & $76.3(60.9)$ & $76.7(61.1)$ & 67.5 \\
\hline I. javanica-099 & 69.7 & 3.47 & $49.3(44.6)$ & $78.3(62.2)$ & $81.1(64.2)$ & 66.8 \\
\hline M. anisopliae-1299 & 64.0 & 3.50 & $77.4(61.6)$ & $82.7(65.4)$ & $86.7(68.6)$ & 67.5 \\
\hline B. bassiana-409 & 64.0 & 6.99 & $20.3(26.8)$ & $62.1(52.0)$ & $78.2(62.2)$ & 63.7 \\
\hline B. bassiana- 4565 & 62.4 & 1.46 & $57.2(49.1)$ & $76.6(61.1)$ & $89.9(71.5)$ & 68.2 \\
\hline B. bassiana- 4511 & 59.3 & 6.53 & $75.0(60.0)$ & $88.6(70.3)$ & $95.1(77.2)$ & 70.3 \\
\hline B. bassiana- 6097 & 51.7 & 4.39 & $23.6(29.1)$ & $64.4(53.4)$ & $81.7(64.7)$ & 60.5 \\
\hline B. bassiana- 4543 & 50.3 & 5.86 & $67.4(55.2)$ & $80.0(63.4)$ & $85.4(67.5)$ & 62.1 \\
\hline $\mathrm{CD}$ at $(p-<0.05)$ & 8.231 & 1.231 & 11.972 & 6.053 & 5.039 & 4.253 \\
\hline Variance & 24.152 & 4.213 & 143.326 & 36.638 & 25.387 & 21.571 \\
\hline
\end{tabular}

a) Experiment was conducted under polyhouse at $33.7-26.7^{\circ} \mathrm{C}$ Max. Mini Temperature and $80.3-68.4 \% \mathrm{RH} .{ }^{b)}$ Mycelial growth diameter was measured (in $\mathrm{mm}$ ) at 10 days post inoculation (DAI) from the Petri plates. ${ }^{c)}$ The spore concentration/mL was measured at 10 DAI using $5 \mathrm{~mm}$ mycelial disc from the Petri plates. ${ }^{d)}$ Figure in parenthesis are arcsign transformed values. ${ }^{e)}$ Biological efficacy index $(\mathrm{BI})=$ mycelia growth $(\mathrm{mm})$, sporulation $($ conidia $\left.1 \times 10^{8}\right)$; nymphal mortality $7 \mathrm{DAI}(\%) ; \mathrm{BI}=37 \times(\mathrm{MG})+13 \times(\mathrm{SP})+50 \times(\mathrm{MO}$ at $7 \mathrm{DAI})$ 
in controlled conditions, while, in field conditions, whitefly mortality ranged from $30-92.2 \%{ }^{38)}$ In previous studies, the selection of EPFs might have been based on either mortality. Here, in this study, we have considered mycelial growth, sporulation, and virulence, which are the most important components for fungal growth, survival, infection, and proliferation. Thus, the results of the present study provide a better understanding for the selection of EPFs.

\subsection{Pathogenicity bioassay and Log dose probit analysis}

In the present investigation, all fungal isolates were pathogenic to whitefly nymphs, and increasing fungal concentrations resulted in increased mortality (Table 2). The $\mathrm{LC}_{50}$ values of EPFs were found to be in a range from $0.2 \times 10^{4}$ to $4.9 \times 10^{6}$ conidia/ $\mathrm{mL}$, while the $\mathrm{LC}_{90}$ values ranged between $1.0 \times 10^{8}$ and $8.7 \times 10^{8}$ conidia/mL at $5 \mathrm{DAI}$. The lowest $\mathrm{LC}_{50}$ values were $0.2 \times 10^{4}$ and $0.5 \times 10^{4}$ conidia/mL, with $\mathrm{Ij}-089$ and $\mathrm{Bb}-4511$, respectively. Similarly, the lowest $\mathrm{LC}_{90}$ values $-1.0 \times 10^{8}$ and $1.0 \times 10^{8}$ conidia/ $\mathrm{mL}$ - were with Ij-089 and Bb-4511, respectively (Table 2). We observed that nymphal mortality also depends upon the virulence and the conidial concentration. Thus, from the present study, it is clear that, to determine the best dose for field application, the lethal concentration that achieves the best results in terms of pest management must be evaluated. The isolates of B. bassiana and I. fumosorosea were reported to be the most virulent against whitefly nymphs (71-86\% mortality within eight days), with $\mathrm{LT}_{50}$ values ranging from three to four DAI with $10^{7}$ conidia/mL (150 conidia/ $\left./ \mathrm{mm}^{2}\right) .{ }^{39)}$ These applications have been successful in cases where environmental conditions of high relative humidity and moderate temperatures are appropriate. ${ }^{40)}$ The average nymphal mortality of $B$. tabaci biotype B at seven DAI can be at the maximum value of $25.7 \%$, and the average mortality at 14 DAI has been reported to vary from $6.1-92.3 \%$ using a melon leaf bioassay method. ${ }^{41)}$ Different isolates of $B$. bassiana a concentration of $10^{7}$ on the fourth instar nymphs gave 3-85\% mortality. ${ }^{42)}$ Thus, the experiment suggests that to further strengthen the selection of EPFs for biocontrol, it is important to consider all of the biological efficacy parameters. Additionally, a study of their pesticide compatibility should be carried out to develop an effective IPM system.

\section{Compatibility study}

\subsection{Mycelial growth inhibition}

All tested insecticides showed varying degrees of potential to inhibit or enhance growth and the conidial production of selected EPFs. The data showed varying responses of EPFs with the chemical and botanical pesticides tested (Supplemental Table S3). Half doses of botanical insecticides, namely neem, pongamia, and castor oils, are less inhibitory to the EPFs than full dose. The growth of EPF strains Ij-089 and Bb-4543 were found to be enhanced with half doses of pongamia oil, as compared to control treatment. Ij-089 also showed enhanced growth with full or half doses of castor oil, and Ij-102 at a half dose, as compared to control and other treatments. Overall, among all of the EPFs evaluated for botanical pesticide compatibility, the EPF strains, namely Ij-089, Ij-102, Bb-4543, and Bb-4565, were found to be comparatively more compatible.

When compared to the effect of chemical insecticides on mycelial growth inhibition, the EPF strains again showed variable

Table 2. Summary of probit analysis of binomial proportion and calculated lethal concentration of dose-mortality response at 5 days post inoculation of whitefly nymphs.

\begin{tabular}{|c|c|c|c|c|c|c|c|c|c|c|}
\hline \multirow{2}{*}{ Spore $\operatorname{conc}^{a)}$} & \multicolumn{10}{|c|}{ Mortality $(\text { Mean } \pm \mathrm{SE})^{b)}$} \\
\hline & $\mathrm{Ij}-089$ & $\mathrm{Ij}-102$ & Fm-83 & $\mathrm{Ij}-099$ & Ma-1299 & Bb-409 & Bb-4565 & Bb-4511 & Bb-6097 & $\mathrm{Bb}-4543$ \\
\hline $10^{4}$ & $74.2 \pm 14.2$ & $52.5 \pm 9.2$ & $45.8 \pm 6.3$ & $27.7 \pm 11.9$ & $48.5 \pm 8.1$ & $14.23 \pm 2.8$ & $64.3 \pm 12.4$ & $52.3 \pm 14.2$ & $15.1 \pm 5.8$ & $22.0 \pm 3.3$ \\
\hline $10^{5}$ & $79.4 \pm 2.2$ & $67.9 \pm 8.2$ & $69.3 \pm 11.2$ & $44.2 \pm 24.4$ & $65.3 \pm 6.4$ & $24.5 \pm 8.9$ & 64.910 .2 & $78.4 \pm 18.2$ & $24.1 \pm 17.1$ & $22.7 \pm 14.6$ \\
\hline $10^{6}$ & $85.6 \pm 7.3$ & $71.0 \pm 4.3$ & $75.8 \pm 8.7$ & $50.0 \pm 12.4$ & $78.7 \pm 5.2$ & $31.21 \pm 11.4$ & $70.5 \pm 7.5$ & $81.4 \pm 17.4$ & $26.8 \pm 20.5$ & $52.1 \pm 6.1$ \\
\hline $10^{7}$ & $86.8 \pm 7.3$ & $74.5 \pm 2.9$ & $78.5 \pm 7.4$ & $72.3 \pm 3.8$ & $80.6 \pm 7.2$ & $51.8 \pm 18.4$ & $77.8 \pm 5.9$ & $85.2 \pm 20.1$ & $51.9 \pm 16.3$ & $69.3 \pm 11.8$ \\
\hline \multirow[t]{2}{*}{$10^{8}$} & $97.1 \pm 2.9$ & $86.6 \pm 4.1$ & $81.9 \pm 4.6$ & $78.5 \pm 0.7$ & $83.6 \pm 6.7$ & $67.21 \pm 14.8$ & $78.9 \pm 4.7$ & $87.1 \pm 18.4$ & $57.7 \pm 8.6$ & $95.8 \pm 4.2$ \\
\hline & \multicolumn{10}{|c|}{ Summary of Probit analysis } \\
\hline $\mathrm{LC}_{50}$ & $0.2 \times 10^{4}$ & $0.6 \times 10^{4}$ & $0.6 \times 10^{5}$ & $1.0 \times 10^{6}$ & $0.6 \times 10^{5}$ & $2.4 \times 10^{6}$ & $0.7 \times 10^{5}$ & $0.5 \times 10^{4}$ & $4.9 \times 10^{6}$ & $1.0 \times 10^{6}$ \\
\hline $95 \% \mathrm{FL}^{c)}$ & $\begin{array}{l}0.1 \times 10^{4}- \\
0.3 \times 10^{4}\end{array}$ & $\begin{array}{l}0.6 \times 10^{4}- \\
0.7 \times 10^{4}\end{array}$ & $\begin{array}{l}0.6 \times 10^{5}- \\
0.7 \times 10^{5}\end{array}$ & $\begin{array}{l}0.9 \times 10^{6}- \\
1.3 \times 10^{6}\end{array}$ & $\begin{array}{c}0.6 \times 10^{5}- \\
0.7 \times 10^{5}\end{array}$ & $\begin{array}{r}1.4 \times 10^{6}- \\
3.5 \times 10^{6}\end{array}$ & $\begin{array}{r}0.6 \times 10^{5}- \\
0.8 \times 10^{5}\end{array}$ & $\begin{array}{l}0.5 \times 10^{4}- \\
0.6 \times 10^{4}\end{array}$ & $\begin{array}{l}0.6 \times 10^{6}- \\
\quad 10.5 \times 10^{6}\end{array}$ & $\begin{array}{r}0.9 \times 10^{6}- \\
1.1 \times 10^{6}\end{array}$ \\
\hline $\mathrm{LC}_{90}$ & $1.0 \times 10^{8}$ & $1.2 \times 10^{8}$ & $1.2 \times 10^{8}$ & $1.7 \times 10^{8}$ & $1.1 \times 10^{8}$ & $4.2 \times 10^{8}$ & $1.8 \times 10^{8}$ & $1.0 \times 10^{8}$ & $8.7 \times 10^{8}$ & $1.6 \times 10^{7}$ \\
\hline $95 \% \mathrm{FL}$ & $\begin{array}{c}0.9 \times 10^{8}- \\
\quad 1.1 \times 10^{8}\end{array}$ & $\begin{array}{c}1.1 \times 10^{8}- \\
1.3 \times 10^{8}\end{array}$ & $\begin{array}{c}1.1 \times 10^{8}- \\
1.3 \times 10^{8}\end{array}$ & $\begin{array}{l}1.4 \times 10^{8}- \\
2.0 \times 10^{8}\end{array}$ & $\begin{array}{c}1.0 \times 10^{8}- \\
1.2 \times 10^{8}\end{array}$ & $\begin{array}{l}2.1 \times 10^{8}- \\
\quad 6.3 \times 10^{8}\end{array}$ & $\begin{array}{c}1.3 \times 10^{8}- \\
2.3 \times 10^{8}\end{array}$ & $\begin{array}{c}0.9 \times 10^{8}- \\
\quad 1.1 \times 10^{8}\end{array}$ & $\begin{array}{l}1.7 \times 10^{8}- \\
\quad 19.2 \times 10^{8}\end{array}$ & $\begin{array}{r}1.4 \times 10^{7}- \\
1.8 \times 10^{7}\end{array}$ \\
\hline Intercept $\pm \mathrm{SE}^{d)}$ & $4.2 \pm 2.1$ & $3.5 \pm 3.6$ & $3.6 \pm 3.6$ & $3.2 \pm 5.3$ & $3.4 \pm 3.6$ & $3.0 \pm 8.5$ & $4.1 \pm 3.2$ & $3.7 \pm 3.0$ & $3.1 \pm 9.8$ & $3.0 \pm 5.1$ \\
\hline Slope \pm SE & $2.5 \pm 0.3$ & $2.7 \pm 0.6$ & $2.6 \pm 0.6$ & $2.4 \pm 0.7$ & $2.8 \pm 0.6$ & $2.1 \pm 0.9$ & $1.9 \pm 0.5$ & $2.7 \pm 0.5$ & $1.9 \pm 1.0$ & $2.8 \pm 0.7$ \\
\hline$\chi^{2 e)}$ & 66.0 & 29.0 & 49.0 & 130.5 & 47.8 & 178.2 & 69.7 & 36.5 & 147.5 & 323.5 \\
\hline$p$-value $\left(\chi^{2}\right)$ & $<0.001$ & $<0.001$ & $<0.001$ & $<0.001$ & $<0.001$ & $<0.001$ & $<0.001$ & $<0.001$ & $<0.001$ & $<0.001$ \\
\hline
\end{tabular}

a) The concentrations presented are conidia/mL . ${ }^{b)}$ Average maximum and minimum \% RH during the experiment 76.7 (50-87) and 58.5 (40-72) during the last week of July and first week of August $2017 .{ }^{c)} \mathrm{FL}=$ fiducial limits; $\left.{ }^{d}\right) \mathrm{SE}=$ standard error of the fungal concentrations. ${ }^{e}$ Likelihood ratio $\chi^{2}$ test statistic indicates a satisfactory goodness-of-fit of empirical data compared to estimated regression line. 
responses against the insecticides tested. Mycelial growth inhibition ranged from -169-94.1\% (Supplemental Table S4). However, the half dose of spiromesifen was found to enhance the mycelial growth of Ma-1299, Ij-089, Bb-6097, Bb-4543, Bb-409, $\mathrm{Ij}-102$, and $\mathrm{Bb}-4565$ better than the control treatment. Similarly, a half dose of flonicamid also enhanced the growth of Bb-409, $\mathrm{Bb}-4543, \mathrm{Ij}-102$, and Bb-4565 better than the control treatment. Moreover, the enhanced growth of EPFs was recorded with a half dose of buprofezin in Bb-6097, Bb-409, and Bb-4543 and a half dose of pyriproxyfen in Bb-4511, Fm-083, and Bb-4543. Overall, the EPFs, namely Ij-089 and Fm -083, were found to be the most compatible with all of the chemical pesticides except triazophos and diafenthiuron, respectively. We found that spiromesifen (tetronic and tetramic acid derivatives-inhibitors of acetyl CoA carboxylase), diafenthiuron (mitrochondrial ATP synthesis inhibitor), buprofezin, pyriproxyfen (insect growth regulators), and flonicamid were comparatively more compatible at their half doses than other chemical pesticides, namely imidacloprid (neonicotinoids), fipronil (phenylpyrazoles), profenophos, and triazophos (organophosphates).

In earlier studies, strains of $B$. bassiana were reported to be highly compatible with the insecticides imidacloprid and spinosad, recording no inhibition of growth, sporulation, or viability. ${ }^{43,44)}$ Similarly, a synergic effect between wettable powder insecticides and $M$. anisopliae ${ }^{24)}$ and a high compatibility of imidacloprid with fipronil for B. bassiana and M. anisopliae have been confirmed by Moino and Alves. ${ }^{22)}$ At doses of 160 and $240 \mathrm{~mL} /$ ha, the insecticide fipronil $(25 \% \mathrm{w} / \mathrm{v})$ was reported to be nondetrimental to $M$. anisopliae mycelial growth and conidiation; thus, it was scored as compatible. ${ }^{45)}$ However, in our present study, this chemical was classified as moderately toxic to Ma-1299. The results of the present study may not be similar to the previous studies because of the difference in the strains and experimental conditions.

Moino and Alves ${ }^{22)}$ previously suggested that there could be two possible reasons for the enhanced growth of EPFs: first, in fungi, as a physiologic mechanism of resistance, insecticides can be metabolized and release compounds that can be used by the fungus as secondary nutrients; second, in a toxic medium, the fungus could be making a reproductive effort, thus increasing conidial production. Another possible reason could be that substances present in the insecticide formulations can also be used directly as a nutrient, which may help increase the vegetative growth and conidial production of the EPFs. However, in the present study, we have observed enhanced mycelial growth in some EPFs; however, corresponding to that, the conidial production was not enhanced.

\subsection{Spore production}

The perusal of the data showed that almost all 12 chemicals showed reductions in conidial production irrespective of the fungal strains, except a few insecticides that were found to enhance the conidial production more than that in the control treatment (Supplemental Table S5). The reduction in conidial production ranged from $-25.6-87.6 \%$ (Supplemental Table S6).
Enhanced conidial production was recorded with a full dose of spiromesifen in Ma-1299, a half dose of spiromesifen in Ij-102, full and half doses of diafenthiuron in Fm-083, and a full dose of castor oil in Bb-4565. When compared to the general spore production, the highest spore production capacity was found to be in Bb-6097 and Fm-083. We compared the effect of all insecticides on individual EPFs. The highest conidial production $\left(10^{7}\right)$ of Ij-99 was recorded with a half dose of spiromesifen. This was followed by a lower dose of neem oil and a lower dose of pongamia oil and flonicamid. The highest conidial production of Ma-1299 was recorded with a full dose of spiromesifen, a full dose of diafenthiuron, and a full dose of pyriproxyfen. Ij-089 produced the highest conidia with a half dose of diafenthiuron, a half dose of spiromesifen, and a full dose of imidacloprid. The highest conidial concentration in Bb-6097 was recorded with half and full doses of flonicamid and a full dose of diafenthiuron. Fm-083 produced higher conidia with half and full doses of diafenthiuron, followed by full and half doses of spiromesifen. Conidiation of Bb-4543 was recorded highest with a half and a full dose of buprofezin and a half dose of diafenthiuron, while $\mathrm{Bb}-4511$ produced more conidia with a half dose of buprofezin, followed by half doses of neem oil and pongamia oil. Ij-102 showed highest conidial production with a full dose and a half dose of spiromesifen and a half dose of neem oil. Bb-4565 showed highest conidial production with a full dose of castor oil and full dose and half dose of profenophos. As the germination of conidia was also evaluated with full and half doses of pesticides in each chemical treatment with respective EPFs, however, we could not observe much difference in the reduction of conidial germination (germination range 96-98\%). Moreover, the present study showed that spore production depends on the resistance and tolerance ability of the EPFs to chemical pesticides.

Furthermore, to reach a comprehensive conclusion regarding the compatibility of EPF strains with test chemicals, the " $T$ " toxicity index was calculated using values of $20 \%$ vegetative growth and $80 \%$ sporulation, which showed the variable responses of EPFs with botanical and chemical pesticides (Table 3). The toxicity index in the present study showed that, among the botanical pesticides, neem oil and pongamia oil were found to be compatible to moderately toxic with all EPFs except Bb-6097, $\mathrm{Bb}-409$, and $\mathrm{Bb}-4543$. Castor oil was found to be toxic to very toxic, except in EPF strains Ij-089, Bb-4565, and Fm-83. Among the chemical insecticides, the insect growth regulators (spiromesifen, pyriproxyfen, buprofezin), flonicamid, and diafenthiuron showed responses that were compatible to moderately toxic. The organophosphate group of pesticides and fipronil were found to be in the toxicity range from compatible to very toxic. Among the EPFs, Ij-089, Bb-4511, and Bb-4565 were found to be compatible with profenophos. Overall, among the EPF strains, Ij089, Ij-102, Ma-1299, and Bb-4511 were found to be the most compatible with full and half doses of the chemical and botanicals tested in the present study. Thus, the compatible EPFs along with these selected pesticides could simultaneously be used for IPM. 
Table 3. "T" values (mean \pm S.E.) and compatibility classification of chemical and botanical insecticides, in relation of fungitoxic effect on selected strains of entomopathogenic fungi.

\begin{tabular}{|c|c|c|c|c|c|c|c|c|c|c|c|c|c|c|c|c|c|}
\hline \multirow{2}{*}{ Treatment ${ }^{a)}$} & \multicolumn{17}{|c|}{ “T” value and compatibility of test chemicals with EPF strains ${ }^{b)}$} \\
\hline & $\mathrm{Ij}-089$ & & $\mathrm{Ij}-099$ & & $\mathrm{Ij}-102$ & & Ma-1299 & Fm-083 & & Bb-6097 & & $\mathrm{Bb}-4511$ & & Bb-4543 & & $\mathrm{Bb}-4565$ & Bb-409 \\
\hline Neem-F & $64.1 \pm 4.5$ & $\mathrm{C}$ & $82.1 \pm 1.3$ & $\mathrm{C}$ & $77.6 \pm 1.2$ & $\mathrm{C}$ & $66.7 \pm 3.1 \quad \mathrm{C}$ & $60.2 \pm 0.7$ & $\mathrm{C}$ & $59.1 \pm 6.6$ & MT & $70.4 \pm 9.2$ & $\mathrm{C}$ & $42.6 \pm 4.4$ & $\mathrm{~T}$ & $52.7 \pm 5.7 \mathrm{MT}$ & $38.0 \pm 2.9$ \\
\hline Neem-H & $47.3 \pm 5.0$ & MT & $69.5 \pm 6.7$ & $\mathrm{C}$ & $65.9 \pm 3.3$ & $3 \mathrm{C}$ & $54.5 \pm 3.6 \mathrm{MT}$ & $45.2 \pm 1.6$ & $\mathrm{~T}$ & $26.9 \pm 1.9$ & VT & $42.9 \pm 4.3$ & $\mathrm{~T}$ & $32.2 \pm 5.4$ & $\mathrm{~T}$ & $37.8 \pm 5.2 \mathrm{~T}$ & $28.2 \pm 2.9 \mathrm{VT}$ \\
\hline Pongamia-F & $68.6 \pm 6.0$ & $\mathrm{C}$ & $79.7 \pm 2.0$ & $\mathrm{C}$ & $70.4 \pm 4.7$ & $7 \mathrm{C}$ & $58.9 \pm 2.9 \mathrm{MT}$ & $63.1 \pm 0.2$ & $\mathrm{C}$ & $64.4 \pm 4.8$ & $\mathrm{C}$ & $79.0 \pm 7.6$ & $\mathrm{C}$ & $43.8 \pm 4.6$ & $\mathrm{~T}$ & $48.5 \pm 6.4 \mathrm{MT}$ & $36.7 \pm 0.8 \quad \mathrm{~T}$ \\
\hline Pongamia-H & $51.6 \pm 4.2$ & MT & $71.4 \pm 6.3$ & $\mathrm{C}$ & $54.7 \pm 3.2$ & $2 \mathrm{MT}$ & $54.1 \pm 3.6 \mathrm{MT}$ & $54.9 \pm 0.9$ & MT & $28.2 \pm 1.9$ & $\mathrm{VT}$ & $64.7 \pm 7.0$ & $\mathrm{C}$ & $41.5 \pm 3.7$ & $\mathrm{~T}$ & $48.5 \pm 5.3 \mathrm{MT}$ & $34.8 \pm 8.3 \quad \mathrm{~T}$ \\
\hline Castor oil-F & $96.3 \pm 8.6$ & $\mathrm{C}$ & $35.0 \pm 4.3$ & $\mathrm{~T}$ & $36.2 \pm 2.4$ & $4 \mathrm{~T}$ & $52.9 \pm 3.9 \mathrm{MT}$ & $49.2 \pm 0.5$ & MT & $35.7 \pm 2.9$ & $\mathrm{~T}$ & $39.4 \pm 5.7$ & $\mathrm{~T}$ & $39.8 \pm 2.4$ & $\mathrm{~T}$ & $96.3 \pm 6.6 \quad \mathrm{C}$ & $29.1 \pm 1.7 \mathrm{VT}$ \\
\hline Castor oil-H & $93.6 \pm 3.7$ & $\mathrm{C}$ & $25.4 \pm 4.0$ & VT & $34.7 \pm 4.8$ & $\mathrm{~T}$ & $39.5 \pm 7.2 \mathrm{~T}$ & $47.9 \pm 5.0$ & MT & $29.9 \pm 1.2$ & VT & $34.3 \pm 0.8$ & $\mathrm{~T}$ & $26.7 \pm 2.7$ & VT & $113.4 \pm 4.3 \mathrm{C}$ & $29.2 \pm 1.6 \mathrm{VT}$ \\
\hline Spiromesifen-F & $92.5 \pm 11.2$ & $\mathrm{C}$ & $86.9 \pm 10.7$ & $\mathrm{C}$ & $104.9 \pm 4.0$ & $0 \mathrm{C}$ & $82.6 \pm 2.6 \quad \mathrm{C}$ & $92.7 \pm 7.1$ & $\mathrm{C}$ & $69.3 \pm 3.3$ & $\mathrm{C}$ & $68.5 \pm 2.2$ & $\mathrm{C}$ & $62.5 \pm 1.2$ & $\mathrm{C}$ & $52.7 \pm 0.9 \mathrm{MT}$ & $56.1 \pm 5.9 \mathrm{MT}$ \\
\hline Spiromesifen-H & $68.5 \pm 9.3$ & $\mathrm{C}$ & $76.4 \pm 8.5$ & $\mathrm{C}$ & $99.4 \pm 5.2$ & $2 \mathrm{C}$ & $103.7 \pm 8.5 \mathrm{C}$ & $65.7 \pm 4.4$ & $\mathrm{C}$ & $67.9 \pm 0.7$ & $\mathrm{C}$ & $58.3 \pm 0.6$ & MT & $65.4 \pm 1.1$ & $\mathrm{C}$ & $39.7 \pm 0.3 \quad \mathrm{~T}$ & $37.0 \pm 0.3 \mathrm{~T}$ \\
\hline Flonicamid-F & $68.9 \pm 9.3$ & $\mathrm{C}$ & $82.0 \pm 8.5$ & $\mathrm{C}$ & $74.4 \pm 5.0$ & $\mathrm{C}$ & $88.4 \pm 6.0$ & $81.1 \pm 6.0$ & $\mathrm{C}$ & $72.5 \pm 0.2$ & $\mathrm{C}$ & $65.0 \pm 3.5$ & $\mathrm{C}$ & $57.4 \pm 1.4$ & MT & $53.1 \pm 0.2 \mathrm{MT}$ & $58.5 \pm 1.3 \mathrm{MT}$ \\
\hline Flonicamid-H & $58.1 \pm 10$ & MT & $61.7 \pm 5.9$ & $\mathrm{C}$ & $52.7 \pm 3.3$ & $3 \mathrm{C}$ & $77.0 \pm 1.1 \quad \mathrm{C}$ & $83.6 \pm 5.6$ & $\mathrm{C}$ & $66.7 \pm 0.9$ & $\mathrm{C}$ & $49.6 \pm 6.4$ & MT & $48.9 \pm 1.5$ & MT & $50.4 \pm 4.3 \mathrm{MT}$ & $53.9 \pm 4.2 \mathrm{MT}$ \\
\hline Diafenthiuron-F & $90.5 \pm 9.6$ & $\mathrm{C}$ & $65.0 \pm 0.4$ & $\mathrm{C}$ & $59.1 \pm 3.4$ & $4 \mathrm{MT}$ & $83.6 \pm 4.5$ & $94.8 \pm 0.2$ & $\mathrm{C}$ & $45.1 \pm 4.7$ & $\mathrm{C}$ & $69.3 \pm 0.6$ & $\mathrm{C}$ & $76.3 \pm 10.9$ & $\mathrm{C}$ & $76.7 \pm 5.4 \quad \mathrm{C}$ & $51.1 \pm 0.6 \mathrm{MT}$ \\
\hline Diafenthiuron-H & $45.5 \pm 4.1$ & MT & $61.9 \pm 0.3$ & $\mathrm{C}$ & $53.9 \pm 3.5$ & $5 \mathrm{MT}$ & $81.9 \pm 4.6 \quad \mathrm{C}$ & $88.3 \pm 0.3$ & $\mathrm{C}$ & $63.9 \pm 1.9$ & $\mathrm{~T}$ & $52.4 \pm 3.9$ & MT & $51.5 \pm 1.6$ & MT & $45.0 \pm 4.5 \mathrm{~T}$ & $49.6 \pm 2.1 \mathrm{MT}$ \\
\hline Buprofezin-F & $70.3 \pm 9.2$ & $\mathrm{C}$ & $62.5 \pm 1.6$ & MT & $61.0 \pm 3.2$ & $2 \mathrm{C}$ & $95.0 \pm 1.6$ & $80.4 \pm 0.7$ & $\mathrm{C}$ & $69.3 \pm 2.0$ & $\mathrm{C}$ & $82.7 \pm 7.5$ & $\mathrm{C}$ & $88.4 \pm 2.6$ & $\mathrm{C}$ & $51.7 \pm 4.8 \mathrm{MT}$ & $85.2 \pm 2.8 \quad \mathrm{C}$ \\
\hline Buprofezin-H & $58 \pm 12.1$ & MT & $50.9 \pm 5.4$ & MT & $67.8 \pm 2.5$ & $5 \mathrm{C}$ & $64.3 \pm 1.2 \quad \mathrm{C}$ & $64.6 \pm 1.2$ & $\mathrm{C}$ & $45.8 \pm 0.4$ & $\mathrm{~T}$ & $71.2 \pm 10.6$ & $\mathrm{C}$ & $78.6 \pm 1.8$ & $\mathrm{C}$ & $48.5 \pm 0.2 \mathrm{MT}$ & $45.6 \pm 4.6 \mathrm{MT}$ \\
\hline Pyriproxifen-F & $67.7 \pm 0.2$ & $\mathrm{C}$ & $65.7 \pm 4.5$ & $\mathrm{C}$ & $71.6 \pm 0.4$ & $4 \quad \mathrm{C}$ & $61.1 \pm 5.7 \quad \mathrm{C}$ & $97.2 \pm 0.3$ & $\mathrm{C}$ & $58.3 \pm 0.4$ & MT & $64.6 \pm 8.4$ & $\mathrm{C}$ & $51.6 \pm 2.3$ & MT & $67.1 \pm 1.3 \mathrm{C}$ & $43.5 \pm 4.0 \quad \mathrm{~T}$ \\
\hline Pyriproxifen-H & $49.7 \pm 7.9$ & MT & $73.4 \pm 14.9$ & $\mathrm{C}$ & $65.1 \pm 2.5$ & $5 \mathrm{C}$ & $76.1 \pm 6.9 \quad \mathrm{C}$ & $77.3 \pm 0.8$ & $\mathrm{C}$ & $54.5 \pm 1.9$ & MT & $47.1 \pm 6.1$ & MT & $41.4 \pm 4.7$ & $\mathrm{~T}$ & $64.8 \pm 2.3 \quad \mathrm{C}$ & $37.2 \pm 4.7 \quad \mathrm{~T}$ \\
\hline Profenophos-F & $66.7 \pm 7.9$ & $\mathrm{C}$ & $30.3 \pm 3.3$ & VT & $42.7 \pm 5.7$ & $7 \mathrm{~T}$ & $60.7 \pm 5.2 \quad \mathrm{C}$ & $55.6 \pm 3.3$ & MT & $34.8 \pm 1.3$ & $\mathrm{~T}$ & $84.9 \pm 10.2$ & $\mathrm{C}$ & $32.3 \pm 5.0$ & $\mathrm{~T}$ & $73.6 \pm 2.9 \quad \mathrm{C}$ & $28.6 \pm 3.7 \mathrm{VT}$ \\
\hline Profenophos-H & $62.8 \pm 2.0$ & $\mathrm{C}$ & $22.9 \pm 0.7$ & VT & $42.3 \pm 6.0$ & $\mathrm{~T}$ & $48.3 \pm 6.0 \mathrm{MT}$ & $50.3 \pm 2.1$ & MT & $31.3 \pm 1.4$ & $\mathrm{~T}$ & $75.9 \pm 9.4$ & $\mathrm{C}$ & $24.9 \pm 5.1$ & VT & $61.3 \pm 1.4 \quad \mathrm{C}$ & $25.8 \pm 0.8 \mathrm{VT}$ \\
\hline Triazophos-F & $36.7 \pm 7.7$ & $\mathrm{~T}$ & $29.4 \pm 5.8$ & VT & $53.1 \pm 5.4$ & $4 \mathrm{MT}$ & $64.6 \pm 4.8 \quad \mathrm{C}$ & $70.4 \pm 4.2$ & $\mathrm{C}$ & $34.6 \pm 2.1$ & $\mathrm{~T}$ & $30.4 \pm 6.3$ & VT & $23.6 \pm 5.5$ & VT & $68.6 \pm 1.1 \quad \mathrm{C}$ & $16.9 \pm 1.2 \mathrm{VT}$ \\
\hline Triazophos-H & $40.4 \pm 2.2$ & $\mathrm{~T}$ & $25.4 \pm 5.7$ & VT & $48.4 \pm 5.7$ & $7 \mathrm{MT}$ & $51.6 \pm 2.3 \mathrm{MT}$ & $64.1 \pm 2.6$ & $\mathrm{C}$ & $33.4 \pm 0.8$ & $\mathrm{~T}$ & $21.5 \pm 5.3$ & VT & $17.6 \pm 5.2$ & VT & $56.4 \pm 7.1 \mathrm{MT}$ & $20.2 \pm 1.6 \mathrm{VT}$ \\
\hline Imidacloprid-F & $81.6 \pm 5.4$ & $\mathrm{C}$ & $47.5 \pm 7.2$ & MT & $36.5 \pm 1.8$ & $3 \mathrm{~T}$ & $52.7 \pm 2.7 \mathrm{MT}$ & $56.8 \pm 3.4$ & MT & $37.7 \pm 1.3$ & $\mathrm{~T}$ & $70.4 \pm 1.0$ & $\mathrm{C}$ & $34.5 \pm 5.7$ & $\mathrm{~T}$ & $85.8 \pm 6.6 \quad \mathrm{C}$ & $29.0 \pm 0.7 \mathrm{VT}$ \\
\hline Imidacloprid-H & $91.8 \pm 2.1$ & $\mathrm{C}$ & $38.1 \pm 5.8$ & $\mathrm{~T}$ & $34.1 \pm 1.9$ & $9 \mathrm{~T}$ & $45.7 \pm 3.8 \mathrm{MT}$ & $58.2 \pm 3.6$ & MT & $28.1 \pm 2.8$ & VT & $60.4 \pm 1.9$ & $\mathrm{C}$ & $37.4 \pm 1.8$ & $\mathrm{~T}$ & $76.4 \pm 6.2 \quad \mathrm{C}$ & $30.4 \pm 0.8 \mathrm{VT}$ \\
\hline Fipronil-F & $47.1 \pm 5.8$ & MT & $32.7 \pm 3.1$ & $\mathrm{~T}$ & $46.5 \pm 1.2$ & $2 \mathrm{MT}$ & $69.3 \pm 1.3 \quad \mathrm{C}$ & $50.5 \pm 3.1$ & MT & $52.3 \pm 1.5$ & MT & $75.7 \pm 1.6$ & $\mathrm{C}$ & $28.1 \pm 3.1$ & VT & $55.5 \pm 6.4 \mathrm{MT}$ & $20.1 \pm 0.2 \mathrm{VT}$ \\
\hline Fipronil-H & $42.0 \pm 5.7$ & $\mathrm{~T}$ & $31.4 \pm 3.1$ & $\mathrm{~T}$ & $33.8 \pm 1.9$ & $9 \mathrm{~T}$ & $53.3 \pm 4.3 \mathrm{MT}$ & $39.0 \pm 2.7$ & $\mathrm{~T}$ & $42.6 \pm 0.6$ & $\mathrm{~T}$ & $59.1 \pm 4.1$ & MT & $22.8 \pm 2.8$ & VT & $53.8 \pm 6.7 \mathrm{MT}$ & $18.7 \pm 0.2 \mathrm{VT}$ \\
\hline Control & 19.89 & & 16.85 & & 10.49 & & 12.57 & 8.96 & & 6.96 & & 16.88 & & 11.70 & & 12.98 & 8.75 \\
\hline $\mathrm{SE}(\mathrm{m})$ & 6.98 & & 5.91 & & 3.68 & & 4.41 & 3.14 & & 2.44 & & 5.92 & & 4.11 & & 4.56 & 3.07 \\
\hline $\mathrm{SE}(\mathrm{d})$ & 9.87 & & 8.36 & & 5.21 & & 6.24 & 4.45 & & 3.46 & & 8.38 & & 5.81 & & 6.44 & 4.34 \\
\hline C.V. & 17.85 & & 17.80 & & 10.67 & & 11.31 & 8.14 & & 8.45 & & 16.53 & & 15.25 & & 12.41 & 13.19 \\
\hline F Cal & 8.51 & & 14.29 & & 31.12 & & 17.32 & 32.71 & & 56.97 & & 9.47 & & 27.25 & & 18.29 & 41.03 \\
\hline
\end{tabular}

${ }^{a)}$ F-Full recommende dose and H-Half dose. ${ }^{b}$ ) Formula proposed by Alves et al. ${ }^{36}$ ) where, $\mathrm{T}=\mathrm{VT} 0$ to 30 (very toxic); $\mathrm{T}-31$ to 45 (toxic); MT 46 to 60 (moderately toxic); C $>60$ (compatible).

We hypothesized that the formula proposed by Alves and Lecuona $^{34)}$ represents an appropriate way to evaluate the effect of the products on pathogens in vitro. These studies have the advantage of exposing EPFs to the maximum level and the action of chemicals, something that could not occur in field conditions. In these conditions, growth inhibition may not be a good indication of other fungicidal effects, such as spore viability. Thus, when the insecticide is compatible in vitro, with strong potential for mycelial growth, conidial production, and germination, there is strong evidence about its selectivity in field conditions. $^{21,23,33)}$ This is due to the fact that conidial germination and infection in insects by ingestion or contact are the most essential components. Additionally, the inoculum survival and dispersal of EPFs in the field is also achieved by conidia. ${ }^{33)}$ Thus, if conidial production and germination inhibition occur, pathogen control efficiency will not be fully achieved, as the pathogen is being applied in an inundative form, together or separately with the IPM components, or if the pathogen is naturally present in plants and contacts the product.

We intend that the fungal strains would show variable responses in their mycelial growth and conidial production, which may be due to the strain as well as the differences in chemical pesticides' composition/active ingredients. Hence, it is clear that the screening of EPFs should not only be based on their virulence, but their compatibility must be evaluated for the effect of chemical pesticides on mycelial growth, conidial production, and germination. Variation was also observed in the toxicity response of EPFs-synergistic, antagonistic, or neutral to insecticides. Previous findings have also reported inconsistent interaction between fungi and insecticides and variation between the strains, ${ }^{46)}$ while working with six isolates of B. bassiana, where the colony growth and germination of one isolate was found to be inhibited by carbaryl. Based on the above findings, strains Ij089, Ij-102, Ma-1299, and Bb-4511, which have the best overall bioefficacy and better pesticide compatibility, could be incorporated in IPM/IRM as a microbial control component. 
Previous studies have shown that chlorpyriphos 20\% EC was less toxic to B. bassiana, while spinosad ( $45 \%$ SC), econeem (1\%), quinalphos (25 EC), acetamprid (20\%), endosulfan (35 EC), and thiodicarb (75 WP) were slightly toxic. Imidacloprid (17.8\% SL) and triazophos (40\% EC) were moderately toxic, and profenophos (50\% EC), indoxacarb (14.5\% EC), and methyldemeton were highly toxic. ${ }^{47)}$ Chlorpyrifos has been found to be a very toxic insecticide for mycelial growth and conidial germination, followed by methomyl, carbamate, and pirate. Flufenoxuron, lufenuron, indoxacarb, and emamectin benzoate were comparatively less toxic to mycelial growth (36.8-48.7\% inhibition) and conidial germination (40.3-49.9\% inhibition) of $M$. anisopliae and P. fumosoroseus. Conversely, methoxyfenozide, bifenthrin, abamectin, and curacron were compatible with significantly lesser growth inhibition (25.2-36.5\%) and conidial germination (27.8-43.7\%) of the fungi. Spinosad was observed to be safe to conidial germination and growth of $M$. anisopliae. ${ }^{48)}$ Faraji et al. ${ }^{49)}$ reported that spinosad was most compatible with B. bassiana and M. anisopliae; however, abamectin, imidacloprid, and deltamethrin were compatible with B. bassiana, and a half concentration of deltamethrin, abamectin, and hexafloron with M. anisopliae. Similarly, James and Elzen and and Singh et al. ${ }^{43,50)}$ recorded that imidacloprid had no negative effect on B. bassiana. Naveen et al. ${ }^{9)}$ evaluated seven Indian field populations of B. tabaci for pesticide susceptibility and found Asia-II-7 (Pusa-Delhi) the most susceptible, while Asia-I (south and central India) and Asia-II-1 (northern India) populations showed significant resistance to selected organophosphates, pyrethroids, and neonicotinoid insecticides.

The results of the present study suggest that lower doses of neem oil, pongamia oil, and insect growth regulators are the safest chemical compounds, which can very well be used with $B$. bassiana, M. anisopliae, I. javanica, and I. javanica in IPM. This combination would give an added advantage where insecticideEPFs mixtures introduce multiple mortality factors against the target pest with insecticide, making the insect physiology weak to a desired degree. That would make it much more susceptible to the attack of the EPFs and also delay the chances of expressing resistance to new insecticides. ${ }^{51)}$ This approach in pest management was explored by Brown et al., ${ }^{52)}$ who found that the combination of imidacloprid and B. bassiana provided greater control of adult tarnished plant bugs in cotton over the use of either of them alone.

\section{Conclusion}

The results of the present study demonstrated that, while screening the bioefficacy of EPFs for a sustainable IPM, a thorough bioefficacy and a compatibility study should be conducted. EPFs are found to have better bioefficacy in terms of insect mortality, along with mycelial growth and sporulation; their compatibility with chemical/botanical insecticides can cause increased stress, immunocompromise, and alteration in insect physiology and behavior. This would improve the performance of selected EPFs in an IPM program, with a better biological component. ${ }^{53)}$
The results of this research suggest that chemical and botanical pesticides are not compatible with all effective EPFs. All strains of B. bassiana and I. javanica showed varying levels of compatibility, with respect to chemicals and their doses. However, EPF strains, viz. Ij- 089, Ij-102, Ma-1299, and Bb-4511, showed better compatibility with full and half doses of all the tested chemicals. These in vitro studies exposed EPFs to the maximum action of the pesticides, which usually does not happen in field conditions. However, the study indicates that the best and the most virulent and compatible EPFs have the maximum likelihood for successful IPM of B. tabaci in cotton fields. Further experiments in field conditions are recommended for evaluating the efficacy of these EPFs in combination with chemical and botanical pesticides.

\section{Acknowledgements}

The authors are thankful to Indian Council for Agricultural ResearchCentral Institute for Cotton Research, Regional Station, Sirsa under Indian Council of Agricultural Research, New Delhi for providing necessary laboratory, field facilities and funding during this research.

\section{References}

1) Anonnymous. All India Coordinated Cotton Improvement Project Report (2017).

2) J. Brown, D. Frohlich and R. Rosell: Annu. Rev. Entomol. 40, 511-534 (1995).

3) D. R. Jones: Eur. J. Plant Pathol. 109, 195-219 (2003).

4) S. Singh, S. Pandher, P. Rathore, A. Sharma, K. Singh and R. K. Gumber: Beltwide Cotton Conferences, New Orleans, LA, pp. 830-835, 2016.

5) J. E. Polston, P. De Barro and L. M. Boykin: Pest Manag. Sci. 70, 1547-1552 (2014).

6) J. C. Palumbo, N. C. Toscano, M. L. Blua and H. A. Yoshida: J. Econ. Entomol. 93, 1688-1694 (2000).

7) B. Picó, M. J. Díez and F. Nuez: Horti. 67, 151-196 (1996).

8) D. Monga (ed.): “Cotton Leaf Curl Virus Diseases." Central Institute for Cotton Research, Regional Station, Sirsa, India, 2014.

9) N. C. Naveen, R. Chaubey, D. Kumar, K. B. Rebijith, R. Rajagopal, B. Subrahmanyam and S. Subramanian: Surf. Sci. Rep. 7, 40634 (2017).

10) K. R. Kranthi: Cotton Statistics and News. No. 04, 2014.

11) K. R. Kranthi, D. R. Jadhav, S. Kranthi, R. R. Wanjari, S. Ali and D. Russell: Crop Prot. 21, 449-460 (2002).

12) M. Basit, S. Saeed, M. A. Saleem, I. Denholm and M. Shah: J. Econ. Entomol. 106, 1414-1422 (2013).

13) C. Longhurst, J. M. Babcock, I. Denholm, K. Gorman, J. D. Thomas and T. C. Sparks: Pest Manag. Sci. 69, 809-813 (2013).

14) A. R. Horowitz and I. Ishaaya: Pest Manag. Sci. 70, 1568-15 (2014).

15) http://cibrc.nic.in/ (Accessed 2 March 2018)

16) L. A. Lacey, S. P. Wright and A. A. Kirk: "Classical Biological Control of Bemisia tabaci in the United States-A Review of Interagency Research and Implementation," eds. by J. Gould, K. Hoelmer and J. Goolsby, Springer, Tokyo, pp. 33-69, 2008.

17) A. C. Scorsetti, C. De Gregorio and C. C. López Lastra: BioControl 53, 787-796 (2008).

18) O. A. Borisade and N. Mahan: Afr. J. Microbiol. Res. 9, 988-1000 (2015).

19) L. A. Lacey, D. Grzywacz, D. I. Shapiro-Ilan, R. Frutos, M. Brown- 
bridge and M. S. Goettel: J. Invertebr. Pathol. 132, 1-41 (2015).

20) M. Faria and S. P. Wraight: Crop Prot. 20, 767-778 (2001).

21) M.R. Faria de and S.P. Wraight: Biol. Control 43, 237-256 (2007).

22) A. Moino Jr. and S. B. Alves: An. Soc. Entomol. Bras. 27, 611-619 (1998).

23) T. E. Anderson, A. E. Hajek, D. W. Roberts, K. Preisler and J. L. Robertson: J. Econ. Entomol. 82, 83-89 (1998).

24) A. Duarte, J. M. Menendez and N. Triguero: Rev. Baracoa 22, 31-39 (1992).

25) A. R. Malo: Rev. Colomb. Entomol. 19, 151-158 (1993).

26) R. A. Humber: "Manual of Techniques in Invertebrate Pathology," ed. by L.A. Lacey, Academic Press, London, pp. 151-187, 2012.

27) T. J. White, T. Bruns, S. Lee and J. W. Taylor: "PCR Protocols: A Guide to Methods and Applications," eds. by M. A. Innis, D. H Gelfand, J. J. Sninsky and T. J. White, New York, Academic Press Inc., pp. 315-322, 1990.

28) X. Huang and A. Madan: Genome Res. 9, 868-877 (1999).

29) S. K. Sain, D. Monga, D. Rishi Kumar: Nagrale and S. Kranthi: Abstr. 7th Asian Cotton Research \& Development Network Meeting, p. 21, 2017.

30) W. S. Abbott: J. Econ. Entomol. 18, 265-267 (1925).

31) D. J. Finney: "Probit Analysis," 3rd Ed., Cambridge University Press, 1971

32) Y. L. Nene and P. N. Thapliyal (eds.): "Fungicides in Plant Disease Control." Oxford \& IBH Publishers, New Delhi, 1997.

33) R. C. de Olivera and P. M. O. J. Neves: Neotrop. Entomol. 33, 353-358 (2004).

34) S. B. Alves and R. E. Lecuona: "Controle Microbiano de Insetos," ed. by S.B. Alves, São Paulo, Fealq. pp. 97-170, 1998.

35) O. P. Sheoran, D. S. Tonk, L. S. Kaushik, R. C. Hasija and R. S. Pannu: "Recent Advances in Information Theory, Statistics and Computer Applications," eds. by D.S. Hooda and R.C. Hasija RC, CCS HAU, Hisar, India, 1998

36) H. N. Zaki: J. Appl. Entomol. 22, 397-399 (1998).
37) A. Karthikeyan and V. Selvanarayanan: Resent Res. Sci. Tech. (Paris) 3, 142-143 (2011).

38) Y. A. Batta: Crop Prot. 22, 415-422 (2003).

39) G. M. Mascarin, N. N. Kobori, E. D. Quintela and I. Delalibera Jr.: Biol. Control 66, 209-218 (2013).

40) D. Chandler, J. B. Heale and A. T. Gillespie: Mycol. Res. 98, 84-388 (1994).

41) S. Vicentini, M. Faria and R. V. M. De Oliveira: Neotrop. Entomol. 30, 97-103 (2001)

42) E. Quesada-Moraga, E. A. Maranhão, P. Valverde-Garcia and C. Santiago-Álvarez: Biol. Control 36, 274-287 (2006).

43) R. R. James and E. W. Elzen: J. Econ. Entomol. 94, 357-361 (2001).

44) S. Bhattacharya, S. Dutta and T. Dhar: Ann. Plant Prot. Sci. 12, 190202 (2004).

$45)$ R. F. T. Ferreira, A. Ferreira, H. F. Prando, F. A. Tcacenco, A. D. Grützmacher and J. F. S. Martins: Cienc. Rural 40, 745-775 (2010).

46) T. E. Anderson and D. W. Roberts: J. Econ. Entomol. 76, 1437-1441 (1983).

47) M. Amutha, J. Gulsar Banu, T. Surulivelu and N. Gopalakrishnan: J. Biopesticides 3, 143-146 (2010).

48) M. R. Asia, M. H. Bashir, M. Afzal, M. Ashfaq and S. T. Sahi: Pak. J. Bot. 42, 4207-4214 (2010).

49) F. Faraji, A. D. Shadmehri and A. Mehrvar: J. Entomol. Soc. Iran 36, 137-146 (2016).

50) R. K. Singh, S. Vats, B. Singh and R. K. Singh: Res. J. Pharm. Biol. Chem. Sci. 5, 837-844 (2014).

51) G. P. Georghiou: "Pest Resistance to Pesticides," eds. by G.P. Georghiou and T. Saito, Plenum Press, New York, 1983.

52) J. Z. Brown, D. C. Steinkraus and N. P. Tugwell: Proc. of the Beltwide Cotton Conference, New Orleans, L.A. National Cotton Council, Memphis, TN, USA, pp. 1302-1305, 1997.

53) S. A. Pelizza, A. C. Scorsetti, M. N. Fogel, S. G. Pacheco-Marino, S. A. Stenglein, M. N. Cabello and C. E. Lange: BioControl 60, 81-91 (2015). 